\title{
Human Motion Change Detection by Hierarchical Gaussian Process Dynamical Model with Particle Filter
}

\author{
Yafeng Yin, Hong Man, Jing Wang, Guang Yang \\ ECE Department, Stevens Institute of Technology, Hoboken, NJ, 07030 \\ \{yyin1,hman,jwang,gyang1\}@stevens.edu
}

\begin{abstract}
Human motion change detection is a challenging task for a surveillance sensor system. Major challenges include complex scenes with a large amount of targets and confusors, and complex motion behaviors of different human objects. Human motion change detection and understanding have been intensively studied over the past decades. In this paper, we present a Hierarchical Gaussian Process Dynamical Model (HGPDM) integrated with particle filter tracker for human motion change detection. Firstly, the high dimensional human motion trajectory training data is projected to the low dimensional latent space with a two-layer hierarchy. The latent space at the leaf node in bottom layer represents a typical human motion trajectory, while the root node in the upper layer controls the interaction and switching among leaf nodes. The trained HGPDM will then be used to classify test object trajectories which are captured by the particle filter tracker. If the motion trajectory is different from the motion in the previous frame, the root node will transfer the motion trajectory to the corresponding leaf node. In addition, HGPDM can be used to predict the next motion state, and provide Gaussian process dynamical samples for the particle filter framework. The experiment results indicate that our framework can accurately track and detect the human motion changes despite of complex motion and occlusion. In addition, the sampling in the hierarchical latent space has greatly improved the efficiency of the particle filter framework.
\end{abstract}

\section{Introduction}

Detection of motion changes in video sequences is very important in the research of computer vision. It can be used to identify suspicious behaviors and irregular motion patterns for surveillance and monitoring. In the real application, due to various environmental conditions, such as illumination, reflection and multiple human objects with occlusion, human motion change detection becomes even more challenging.

The assumption of our work is that normal human is prone to having similar motion trajectory in a specific location, while sudden motion change usually implies suspicious behaviors. If we can learn these similar trajectories in advance, then they can be used for motion trajectory classification and motion change detection. From the learning perspective, previous motion change detection algorithms can be divided into two categories: non-knowledge based detection and knowledge based detection. In the nonknowledge based motion detection, human motion was recognized by statistical methods $[18,21]$, while in the knowledge base motion detection, human motion change is identified by pre-defined patterns [6]. In this paper, we proposed a Hierarchical Gaussian Process Dynamical Model integrated particle filter for human motion change detection. Our framework takes the advantage of both afrontmentioned approaches to formulate a framework combining particle filter tracking and trained HGPDM for classification.

During the training phase, we extract the human motion data from the KTH Action dataset [1], which includes running and walking videos of different people. The walking and running motion trajectories are extracted manually and projected to the leaf nodes of hierarchical latent space Gaussian Process Dynamic Model. After jointly optimization, the root node represents the interaction and switching of leaf nodes, while the leaf nodes denote different types of motion trajectory motion. The learned motion model are then used to classify test object trajectories, predict the next motion state, and provide Gaussian process dynamical samples for the particle filter framework. We test our change detection framework in the video data set used in [7] and the IDIAP data set used in [17]. The experimental results indicate that our framework can detect each motion change correctly.

The major novelty of this paper is that we proposed a Hierarchical Gaussian Process Dynamical Model to learn the prior motion information for motion pattern change detection. The hierarchical structure can address complex human motion as the root node control the interaction between the 
leaf nodes. This paper extends the GPDM in [20] to Hierarchical GPDM and used it for motion pattern recognition. On one hand, the particle filter is capable of tracking nonlinear and non Gaussian human motion. This allows HGPDM to utilize the captured human motion data for classification and change detection over time. On the other hand, the hierarchical structure of GPDM insures the finding of the most similar motion pattern in the leaf nodes. Therefore the sampling in the pre-learned GPDM model improves the efficiency of the particle filter framework.

In contrast to Gaussian Process Dynamical Model used in $[11,13,20]$, we focus on the global human motion rather the local motion of human body parts. Therefore instead of using 3D motion database, we select 2D motion data of multiple human objects to construct a high dimensional training data set, and train our HGPDM. In addition, our framework can address the switching between different motion patterns, which is very critical for change detection of human with complex motion.

Our HGPDM is inspired by the Hierarchical Gaussian Process Latent Variable Model (HGPLVM) in [9]. Neil Lawrence applied Hierarchical GPLVM for modeling the human interaction. Our approach is different from Neil Lawrence's work in two ways: Firstly, we extend the GPLVM to GPDM in the leaf nodes, which gives a compact representation for the joint distribution of observed temporal data in the latent space. In addition, the latent space in root node is optimized after leaf nodes instead of jointly optimizing all the nodes in [9]. In other words, the root node in our framework is a classifier controlling all the switching between leaf nodes.

This paper is organized as follows: Section 2 reviews the related work on the human motion change detection, Hierarchical Gaussian Process Dynamical Model and particle filter for multiple-target tracking. The structure of HGPDM with particle filter is proposed at Section 3. Section 4 describes the detailed training and testing process of HGPDM with particle filter. All the experimental results and comparison are shown in the Section 5. Finally, conclusion and future work is summarized in the Section 6.

\section{Related Work}

Human motion change detection is usually used for action recognition and suspicious behavior analysis [12]. It has attracted much attention in recently years. Many previous works used segmentation and tracking for human motion detection [4, 10]. O. Boiman [3] proposed a probabilistic graphical model for detecting irregularities in the video. N. Kiryati et al.[7] extracted the motion features from videos, and used a pre-trained motion model to classify different human motion. The abnormal human motion pattern relied on the trained motion trajectories. J. Sherrah [16] presented a platform (VIGOUR) for tracking and recognition activities of multiple people. The system can track up to three people and recognize their gestures. However, most previous methods cannot handle human motion change detection with multiple people, due to the increasing computational complexity with multiple targets tracking.

Gaussian Process Latent Variable Model [8] (GPLVM) developed by Neil Lawrence in 2006 provided a probabilistic mapping from high-dimensional observation data to lowdimensional latent space, which represented the joint distribution of observation data. Compared to other dimension reduction algorithms, such as LLE [15] and ISOMAP [19], GPLVM has the advantage to provide the posterior probability of the projected observation space. J. Wang et al. introduced Markov dynamics in latent variable state transitions lending Gaussian Process Latent Variable Model to handle time series data. Their proposed framework can robustly track human body motion and pose dynamics [20]. Leonid et al. proposed a Gaussian process annealing particle filter based method to perform 3D target tracking by exploring color histogram features [13], while he focused on pose reconstruction rather than human trajectory tracking. A real time body particle tracking framework introduced by Hou [5] to capture human motion. However, he aimed to track complex motion of one target and used the motion data for the pose estimation. Neil Lawrence extended the GPLVM to hierarchical GPLVM in [9], in which he used the hieratical GPLVM for modeling the interaction of multiple subjects.

\section{Proposed Framework}

The proposed method is aimed to learn a general human motion trajectory model for multiple human motion change detection. The pre-trained model can robustly detect different human motion change, and reduce the computational complexity as well as improve the robustness of particle tracking framework. The flowchart of our framework is shown in the Figure 1.

The basic procedure of the proposed Particle Filter with Hierarchical Gaussian Process Dynamical Model is described as follows.

1. Creating Hierarchical GPDM: The leaf node of Hierarchical GPDM is created on the basis of the trajectory training data sets, i.e. coordinate difference values, while the top node is created on the basis of dependence of leaf nodes. The learning model parameters include $\Gamma=\left\{Y^{T}, X^{T}, \bar{\alpha}, \bar{\beta}, W\right\}$, where $Y^{T}$ is the training observation data set, $X^{T}$ is the corresponding latent variable sets, $\bar{\alpha}$ and $\bar{\beta}$ are hyperparameters, and $W$ is a scale parameter.

2. Jointly initializing the model parameters: The three nodes of latent variable sets and parameters 


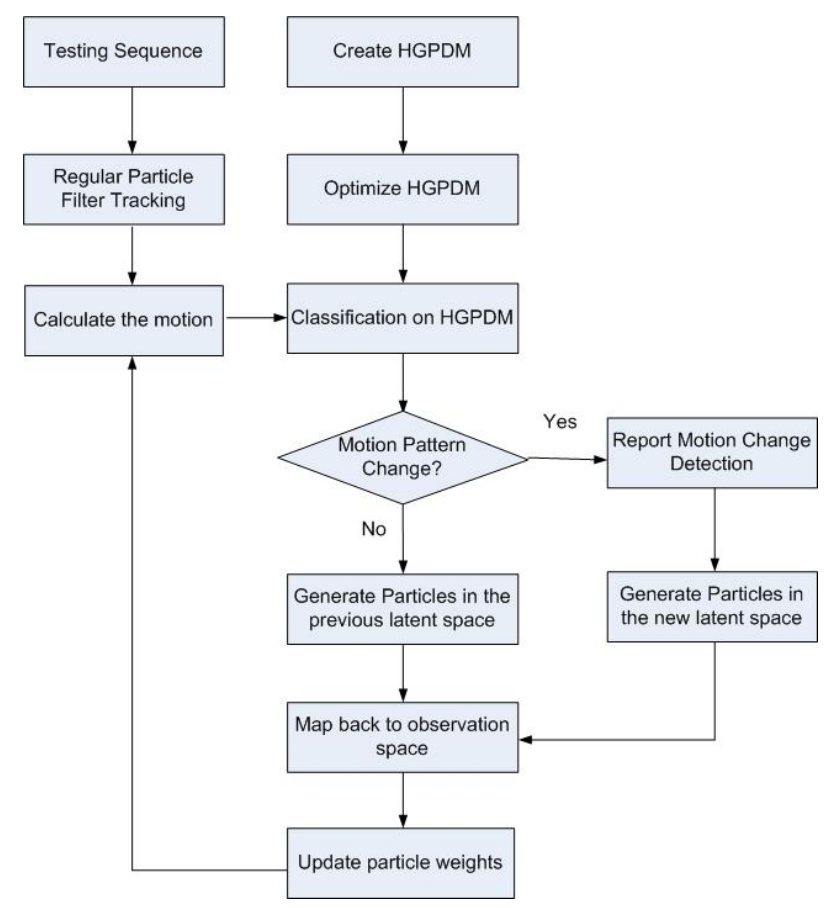

Figure 1. Human motion detection and tracking framework

$\left\{X^{T}, \bar{\alpha}, \bar{\beta}\right\}$ are obtained by minimizing the negative $\log$-posterior function $-\ln p\left(X^{T}, \bar{\alpha}, \bar{\beta}, W \mid Y^{T}\right)$ of the unknown parameters $\left\{X^{T}, \bar{\alpha}, \bar{\beta}, W\right\}$ with scaled conjugate gradient ( $\mathrm{SCG}$ ) on the training datasets.

3. Initializing the particle filter framework: The prior probability is derived on the basis of the created model. In this step, target templates are obtained from the previous frames as reference images for similarity calculation in the later stage.

After initializing the targets' position, each target will be tracked by the regular particle filter in the first five frames. Then test observation motion pattern data is calculated and projected to the latent coordinate system on the top node of HGPDM by using probabilistic principal component analysis (PPCA).

4. Latent space motion classification and change detection: After projecting the current motion data into the latent space, the top node will determine which motion pattern in the leaf node that the current motion belongs to. If the motion pattern is not consistent with the previous one, the motion change is reported and the latent space will switch to the new leaf node

5. Leaf node latent space particle sampling and Predicting: Particles are generated in the latent space of leaf node GPDM to infer the likely coordinate change value $\left(\Delta x_{i}, \Delta y_{i}\right)$.

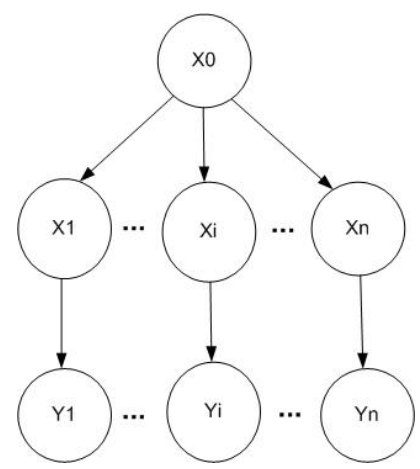

Figure 2. A Hierarchical Gaussian Process Dynamical Model. The root node $X_{0}$ controls all the interactions between the latent space of leaf nodes in $X_{1}, \ldots X_{n} . Y_{1}, . . Y_{n}$ denotes the observation data associated with $X_{1}, \ldots X_{n}$

6. Determining probabilistic mapping from the latent space to observation space: The log posterior probability of the coordinate difference values of the test data is maximized to find the best mapping in the training data sets of the observation space.

In addition, the most likely coordinate change value $\left(\Delta x_{i}, \Delta y_{i}\right)$ is used for predicting the next motion.

7. Updating the weights: In the next frame, the similarity between the template's corresponding appearance model and the cropped region centered on the particle is calculated to determine the weights $w_{i}$, and the most likely location $\left(\hat{x}_{t+1}, \hat{y}_{t+1}\right)$ of the corresponding target, as well as to decide whether resampling is necessary or not.

8. Repeat Step 4 - 7.

\section{HGPDM with Particle Filter}

The reason that we used Hierarchical Gaussian Process Dynamical Model is that the root node can control all the interactions and switchings between each leaf nodes. Therefore it can model more complex motion. Its structure can be described in Figure 2.

\subsection{Human Motion Training by HGPDM}

\subsubsection{Extract Human Motion Trajectory Data}

During the training phase, we manually extracted the motion data from the KTH motion database [1]. At each frame of a walking cycle, we select a point at the central location of the human body. The coordinate difference between two consecutive frames forms a motion vector. Supposing the total frame number is 30 , the motion data of one person is 30 by 2 dimension. In our training set, we choose 20 different people, each walks at a different direction. The total training data is then 30 by 40 dimensional. In this paper, we 
just select two typical motion trajectories in the KTH motion database[1], walking and running, to demonstrate the performance of our proposed framework. In the walking and running categories, we extract different human objects moving at different directions.

\subsubsection{Establishing Trajectory Learning Model On The Leaf Node}

GPDM is applied to learn the specific trajectories of moving human. The probability density function of latent variable $X$ and the observation variable $Y$ are defined by the following equations,

$P\left(Y_{k} \mid X_{k}\right)=\frac{|W|^{N}}{\sqrt{\left(2 \pi^{N D}\left|K_{Y}\right|^{D}\right)}} \exp \left(-\frac{1}{2} \operatorname{tr}\left(K_{Y}^{-1} Y W^{2} Y^{T}\right)\right)$

where $W$ is the hyperparameter, $N$ is the number of $Y$ sequences, $D$ is the data dimension of $Y, K_{Y}$ is the kernel function.

In our study, RBF kernel given by the following equation is employed for HGPDM model,

$$
k_{Y}\left(x, x^{\prime}\right)=\exp \left(-\frac{\gamma}{2}\left\|x-x^{\prime}\right\|^{2}\right)+\beta^{-1} \delta_{X, X^{\prime}}
$$

where $x$ and $x^{\prime}$ are any latent variables in the latent space, $\gamma$ controls the width of the kernel, $\beta^{-1}$ is the variance of the noise.

Given a specific surveillance environment, certain patterns may be observed and worth exploring for future inferences. To initialize the latent coordinate, the $d$ (dimensionality of the latent space) principal directions of the latent coordinates is determined by deploying probabilistic principal component analysis on the mean-subtracted training dataset $Y^{T}$, i.e. $Y^{T}-\overline{Y^{T}}$. Given $Y^{T}$, the learning parameters are estimated by minimizing the negative-log-posterior using scaled conjugate gradient (SCG) [14].

\subsubsection{Optimizing The Hierarchical Gaussian Process Dynamical Model}

As the structure described in Figure 2, $Y_{1}$ and $Y_{2}$ denote the high dimensional multiple human walking and running data, $X_{1}$ and $X_{2}$ represent the corresponding latent space in the leaf nodes. by

The joint probability distribution of $Y_{1}$ and $Y_{2}$ is given

$$
\begin{aligned}
P\left(Y_{1}, Y_{2}\right) & =\int p\left(Y_{1} \mid X_{1}\right) \times \int p\left(Y_{2} \mid X_{2}\right) \ldots \\
& \times \int p\left(X_{0} \mid X_{1}, X_{2}\right) d X_{3} d X_{2} d X_{1}
\end{aligned}
$$

where each conditional distribution is given by Gaussian Process. The major advantage of the GPDM is that each training data is associated with a likelihood in the latent space. We then use the MAP method to find all the values of latent variables. For this simple model, we are trying to optimize the parameters by maximizing the following equation,

$$
\begin{aligned}
\operatorname{logp}\left(X_{0}, X_{1} X_{2} \mid Y_{1}, Y_{2}\right) & =\operatorname{logp}\left(Y_{1} \mid X_{1}\right) \ldots \\
& \times \log p\left(Y_{2} \mid X_{2}\right) \ldots \\
& \times \operatorname{logp}\left(X_{0} \mid X_{1}, X_{2}\right)
\end{aligned}
$$

The training process for optimizing the Hierarchical Gaussian Process Dynamical Model is described as follows:

1. Initial each leaf node: Project the walking and running training data to $\left(X_{1}, X_{2}\right)$ through probabilistic principle component analysis

2. Initial root node: Initialize the root's latent variable through probabilistic principle component analysis and its dependence of $\left(X_{1}, X_{2}\right)$

3. Jointly optimize the parameters of each Gaussian Process Dynamical Model Optimize jointly for the kernel matrix of each GPDM

Once the latent space has been optimized, we applied supervised clustering at the top latent space to group the training data to the leaf node. Supposing we have N leaf nodes, and $Y=\left\{y_{i}, i=1 \ldots M\right\}$. Each leaf node is associated with mean $\mu_{j}$ and variance $\sigma_{j}$, and we assign each training $y_{i}$ to the leaf node by

$$
\hat{X}_{y_{i}}=\arg \max _{j=1,2, \ldots, N} p\left(x^{\prime} \mid \mu_{j}, \sigma_{j}\right), i=1,2, \ldots, M
$$

One example of the learned running latent space in the leaf node is shown in the Figure 3.

\subsection{HGPDM With Particle Filter Testing}

After jointly optimizing the HGPDM, the trained HGPDM can be used to identify the human motion trajectories captured by particle filter tracker. In the meantime, the classified motion pattern provides the most similar motion trajectory for efficient particle sampling. The process of our framework is described as follows.

\subsubsection{Initializing The Particle Filter Framework}

A particle filter is a Monte Carlo method for nonlinear, non-Gaussian models, which approximates continuous probability density function by using large number of 
samples. In our framework, histogram was used as appearance modeling for its simplicity and efficiency. The RGB histogram of the template and the image region under consideration are obtained respectively. The likelihood $P\left(Z_{t} \mid k_{t}, \hat{Y}_{t}\right)$ is defined to be proportional to the similarity between the histogram of the template and the candidate, and is measured by Bhattacharya distance.

At this step, target templates are obtained by the using background subtraction. The obtained target templates will be used for similarity calculation in the testing stage.

After initializing the targets' position for the first frame, each target will be tracked by using regular particle filter in the first five frames. This is based on the assumption that the human motion does not change at the very beginning. Then test observation motion data of the first five frames is calculated and projected to the latent coordinate system on the top node of HGPDM.

\subsubsection{Latent Space Motion Classification and Change Detection}

Since HGPDM was constructed in the latent space, at the beginning of the test process, the target observation data of first five frames has to be projected to the same 2dimensional latent space in order to be compared to the upper level of trained HGPDM. The purpose of projecting the test data from the observation space to the latent space is to initialize the testing data in the latent space and obtain a compact representation of the similar motion patterns in the training data set. This projection is achieved by using probabilistic principal component analysis (PPCA), same as the first stage in HGPDM learning. The feature vector of each frame contains the coordinate change values for every target being tracked in that frame. For $n$ targets, the feature vector will contain $n \times 2$ pairs of coordinate change values. The PPCA projection will reduce this $n \times 2$ dimensional feature vector to a $1 \times 2$ latent space vector. After projecting test motion data from observation to latent space, the upper level of hierarchical GPDM will be used to identify the most similar motion patterns in the leaf node. The classification algorithm in the HGPDM is shown as below:

1. Select top $K$ most likely latent variables $x_{i}$ in the root node by using equation,

$$
x_{i}=\arg \max _{x \in I} K\left(p\left(y^{\prime} \mid x\right)\right), i=1,2, \ldots, K
$$

2. Compute the relative normalized probability as :

$$
\pi_{i}=\frac{p\left(y^{\prime} \mid x_{i}\right)}{\sum_{i=1}^{K} p\left(y^{\prime} \mid x_{i}\right)}
$$

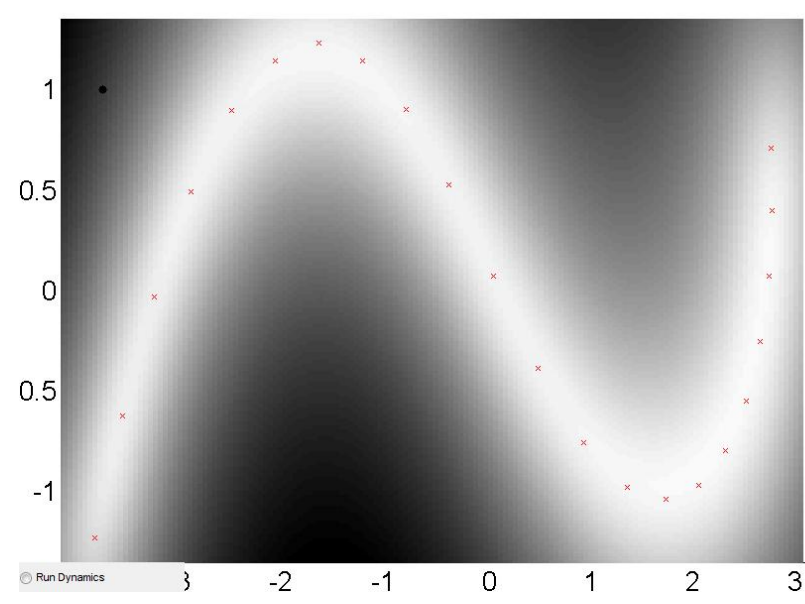

Figure 3. Trained Running Latent Space

\section{Establish the latent variable $x^{\prime}$ as}

$$
x^{\prime}=\sum_{i=1}^{n} \pi_{i} x_{i}
$$

4. Determine the corresponding latent space in the leaf node $\hat{X}$ as:

$$
\hat{X}=\arg \max _{j=1,2, \ldots, N} p\left(x^{\prime} \mid \mu_{j}, \sigma_{j}\right), i=1,2, \ldots, K
$$

At each frame, once the human motion pattern is classified to a different category by equation (9), the system does not immediately transfer the latent space to the corresponding one. If the motion pattern keeps to be identified as another category for five consecutive frames, then the sampling latent space is switched from one leaf node to the other one, and the human motion change is reported to the system. Otherwise the temporary human motion change is consider as noise and the particle filter framework will still perform sampling in the latent space which is determined in the last frame. The next possible position is predicted by determining the most similar trajectory pattern in the leaf node and using the corresponding position change value plus noise.

\subsubsection{Particle Filter Tracking and Update}

After determining the general categories of the human motion pattern in the root node of HGPDM, we propagate the particles in the latent space of leaf node. The next possible position is predicted by determining the most similar trajectory pattern in the training database and using the corresponding position change value plus noise. The number of particles are reduced from over one hundred to about twenty by deriving the posterior distribution over latent functions. Each point on this 2D latent space in the Figure 3 is a projection of a feature vector representing 20 training targets, 
i.e. 20 pairs of coordinate change values. The gray scale intensity represents the precision of mapping from the observation space to the latent space, and the lighter the pixel appears, the higher the precision of mapping is.

Thereafter, the latent variables are mapped in a probabilistic way to the location difference data in the observation space. Estimation maximization (EM) approach is employed to determine the most likely observation coordinates in the observation space after the distribution is derived.

The non-decreasing log posterior probability of the test data is given by equation (1). $K_{Y}$ is a kernel matrix defined by a RBF kernel function given by equation (2). The log posterior probability is maximized to search for the most probable correspondence on the training datasets. The corresponding trajectory pattern is then selected for predicting the following motion.

The weights of the particles are updated in terms of the likelihood estimation based on the appearance model. The importance weight equation is given by

$$
\begin{aligned}
P\left(\hat{Y}_{t} \mid Z_{t}, k_{t}\right) & =\frac{P\left(Z_{t} \mid k_{t}, \hat{Y}_{t}\right) P\left(k_{t}, \hat{Y}_{t}\right)}{P\left(Z_{t}\right)} \\
w_{t} & \propto P\left(Z_{t} \mid k_{t}, \hat{Y}_{t}\right) P\left(k_{t}, \hat{Y}_{t}\right)
\end{aligned}
$$

where $\hat{Y}_{t}$ is the estimation data, $Z_{t}$ is the observation data, $k_{t}$ is the identity of the target, and $w_{t}$ is the weight of a particle.

\section{Experimental Results And Discussion}

The proposed HGPDM particle filter framework was implemented using MATLAB running on a desktop of $2.33 \mathrm{GHz}$ Intel Core 2 Duo CPU PC with $2 \mathrm{~GB}$ memory and tested on the IDIAP datasets used in [17] and the data set used in [7]. Neil Lawrence's Gaussian process softwares provide the related GPDM functions for conducting simulations [2].

The first testing sequence extracted from the IDIAP data set contains two targets and one of them runs after the other one from left to right. The motion data is captured by the particle filter and classified in the HGPDM. Human motion change is reported at the 58th frame. Figure 4 shows the corresponding frame sampled from the tracking results and Figure 5 showed the posterior probability of left target' motion belonged to running GPDM latent space. According to Figure 5 , the posterior probability is higher than 0.5 before the 58th frame, while lower than 0.5 after the 58th frame. This means that the motion trajectory is switched from running to walking in the HGPDM near the 58th frame. The sampling frames in the Figure 4 verifies the human motion change is correctly detected. It need to be noticed that there are several sparks in the posterior probability curve due to the noisy motion data captured by the particle filter.
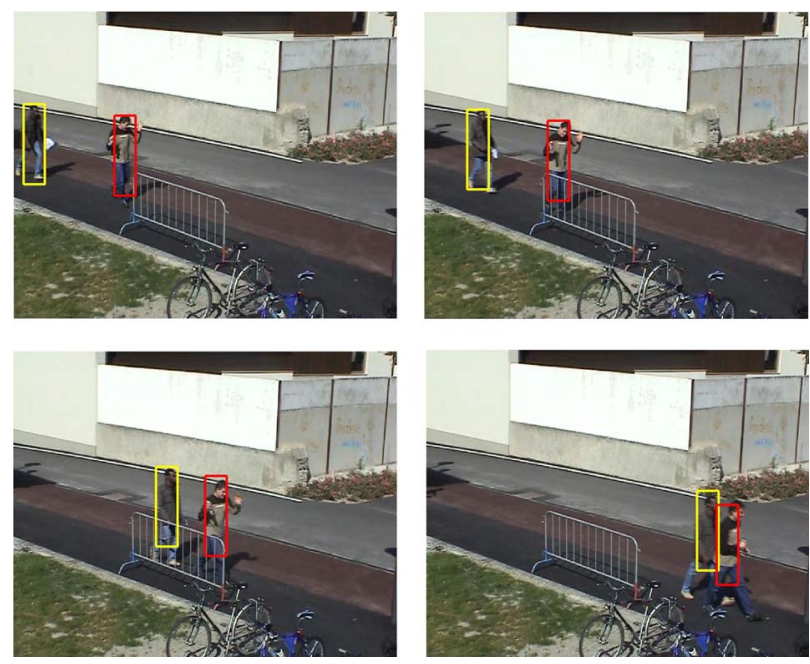

Figure 4. Sampling frames of $3,19,58,98$ in the first testing sequence

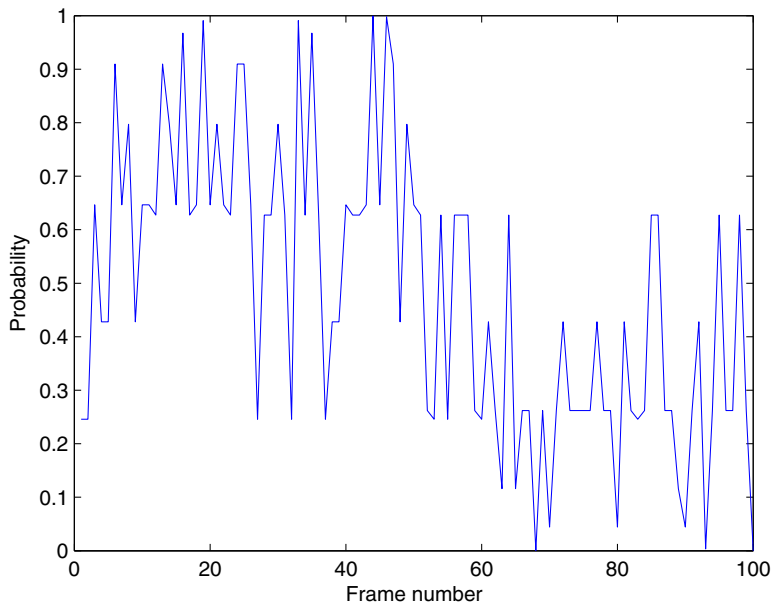

Figure 5. Posterior probability of running motion change

The second sequence contains four people with occlusion and complex motion patterns. The person with blue bounding box firstly walked to the road center, then circled around the bike ramp in the middle of the road. Both the direction and the velocity were varied at this circle motion. Motion change are reported at the 42th and 129th frame respectively. Figure 6 shows the posterior probability of motion change. The posterior probability is below 0.5 near the 42th and 129th frame, as the person made a turn near this two frames respectively. The sampling frames in Figure 7 indicate the motion change is correctly detected.

we apply our system on the data set used in [7], and compare the human motion change detection results with the abnormal motion recognition. The experimental results indicate that our system can detect all the motion change correctly, which are corresponding to the abnormal motion 


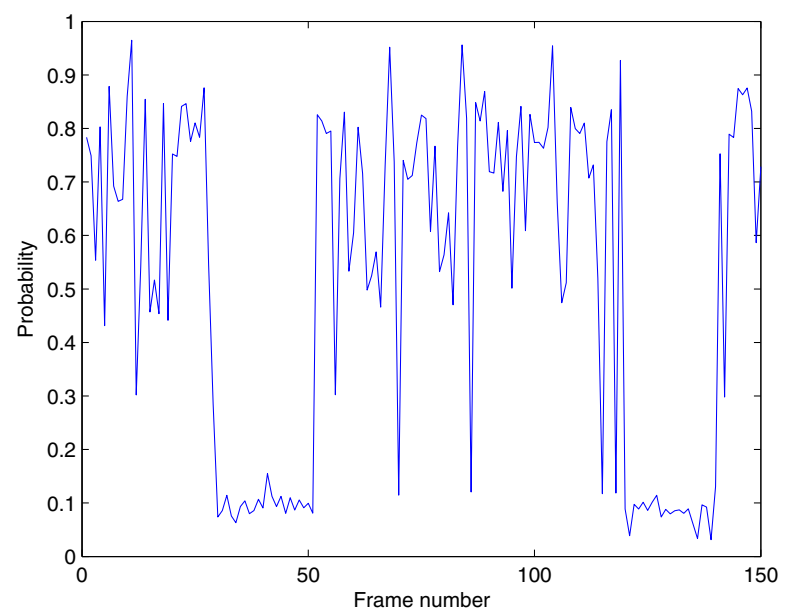

Figure 6. Posterior probability of circle motion
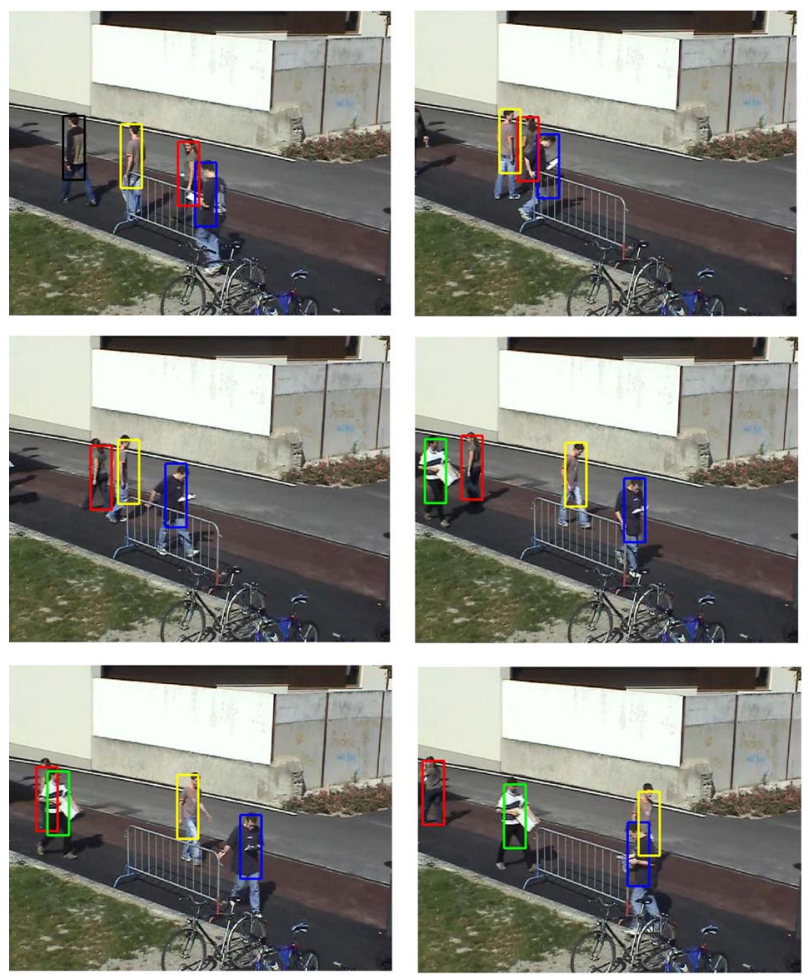

Figure 7. Sampling frames of $1,42,65,87,129,150$ in the second testing sequence

defined in the [7]. One of the testing sequences shown in Figure 8 was defined as jumping episode in [7]. The motion change detection of left target are reported at 18th and 60th frame. According to Figure 8, the woman stopped from running near frame 9 and began to walk after jumping toward the man near frame 60. Our proposed system can capture the motion change successfully through the switching between different latent space nodes.
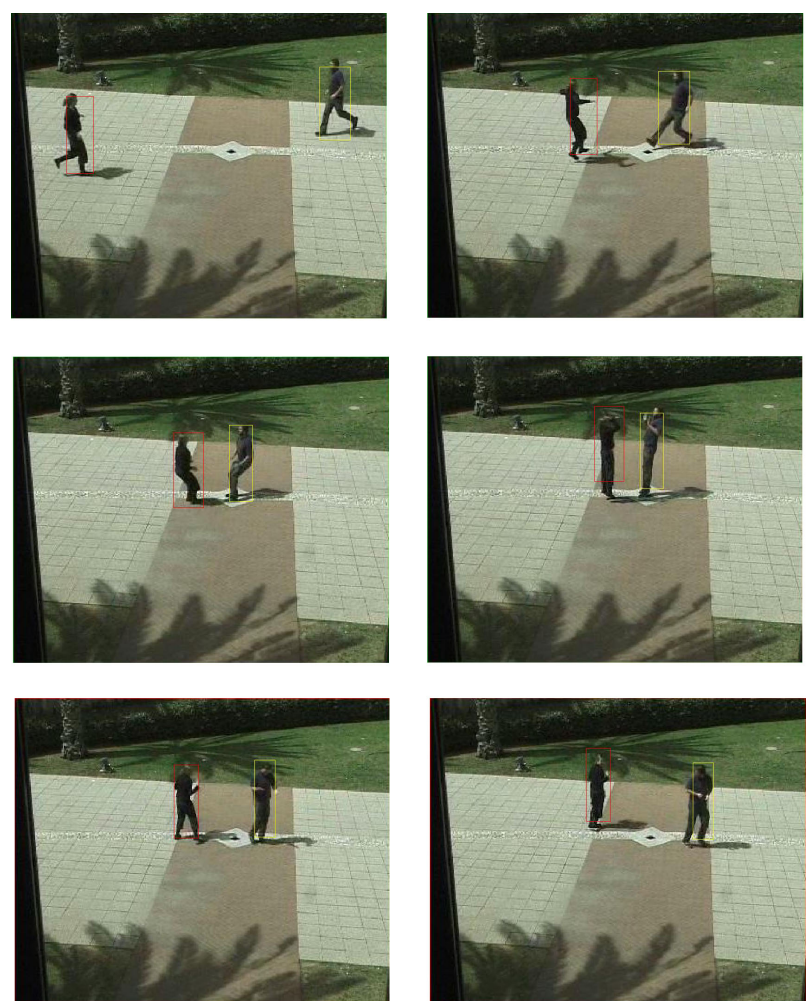

Figure 8 . Sampling frames of $1,18,25,33,60,85$ in the third testing sequence

As all the abnormal motion patterns in [7] were predefined, their system can only detect all the trained trajectories. While we propose a general system for detecting the motion change of tracked targets over time. The detected motion change can be used for suspicious behavior analysis in the surveillance system. Moreover, our system can track up to five targets and detect their motion changes simultaneously, which can provide the group human behavior analysis.

\section{Conclusions and Future Work}

We proposed a Hierarchical Gaussian Process Dynamical Model with particle filter for human motion change detection in the surveillance systems. The proposed system can track up to five targets simultaneously and detect their motion change over time in various visual environment. HGPDM and particle filter help each other for motion change detection. The particle filter framework can accurately capture unexpected, non-linear, and non-Gaussian motion for motion classification in the HGPDM. The pretrained HGPDM provides the most similar motion trajectory for particle filter sampling and tracking, which greatly reduces the particle number. 20 particles are sufficient in our framework to correctly and robustly track each target.

In this paper, we use IDIAP data set and abnormal mo- 
tion data set in [7] to demonstrate the efficiency and accuracy of our proposed framework. The test results indicate that our framework can correctly detect each motion change and robustly track multiple targets with complex motion at the same time.

Because the definition of abnormal motion pattern is different in different applications, we are trying to learn a general motion trajectory model for motion change detection rather abnormal motion detection. The detected motion changes by our framework can be used for suspicious behavior analysis and irregular motion detection. It need to be noted that due to different scales and different directions of viewpoints, the human motion trajectory extracted from images may be different from each other. Therefore the performance our framework may vary with different application.

In the future, our framework can be extended to group human behavior analysis or $3 \mathrm{D}$ multiple human motion modeling.

\section{Acknowledgments}

This work is supported in part by US ARMY RDECOMARDEC under contract W15QKN-05-D-0011, Task Order 56. The authors are also truly grateful to Dr. Kevin Smith for his assistance in providing us the IDIAP test data for our study.

\section{References}

[1] KTH action database. http://www.nada.kth.se/cvap/actions. $1,3,4$

[2] Neil lawrence gaussian process software. http://www.cs.man.ac.uk/ neill/software.html. 6

[3] O. Boiman and M. Irani. Detecting irregularities in images and in video. In Proceedings of International Conference on Computer Vision, 2005. 2

[4] I. Haritaoglu, D. Harwood, and L. S. David. Real-time surveillance of people and their activities. 8(22):809-830, August 2000. 2

[5] S. Hou, A. Galata, F. Caillette, N. Thacker, and P. Bromiley. Real-time body tracking using a gaussian process latent variable model. In International Conference on Computer Vision, 2007. 2

[6] Y. Ivanov and A. Bobick. Recognition of multi-agent interaction in video surveillance. In Proceedings of International Conference on Computer Vision, 1999. 1

[7] N. Kiryati, T. Raviv, Y. Ivanchenko, and S. Rochel. Realtime abnormal motion detection in surveillance video. In Proceedings of International Conference on Pattern Recognition, 2008. 1, 2, 6, 7, 8

[8] N. Lawrence. Probabilistic non-linear principal component analysis with gaussian process latent variable models. 2005. 2

[9] N. D. Lawrence and A. J. Moore. Hierarchical gaussian process latent variable models. In International Conference on Machine Learning, 2007. 2
[10] G. Medioni, R. Nevatia, and I. Cohen. Event detection and analysis from video streams. 2001. IEEE Transactions on Pattern Analysis and Machine Intelligence, volume 23, pages 873-889. 2

[11] D. J. F. R. Urtasun and P. Fua. 3D people tracking with gaussian process dynamical models. 2006. 2

[12] S. Rao and P. Sastry. Abnormal activity detection in video sequences using learnt probability densities. In TENCONO3, pages 369-372, volume 1, 2003. 2

[13] L. Raskin, E. Rivlin, and M. Rudzsky. Using gaussian process annealing particle filter for 3d human tracking. 2007. EURASIP Journal on Advances in Signal Processing. 2

[14] M. Riedmiller and H. Braun. Rprop- a fast adaptive learning algorithm. In Proc. of 7th Int'l Symp. on Computer and Information Sciences (ISCIS VII), 1992. 4

[15] S. Roweis and L. Saul. Nonlinear dimensionality reduction by locally linear embedding. Science, 290:2323-2326, 2000. 2

[16] J. Sherrah and S. Gong. Vigour: A system for tracking and recognition of multiple people and their activities. In Proceedings of International Conference on Pattern Recognition, pages Vol I: 179-182, 2000. 2

[17] K. Smith, D. Gatica Perez, and J. Odobez. Using particles to track varying numbers of interacting people. In IEEE Conference on Computer Vision and Pattern Recognition, 2005. 1,6

[18] C. Stauffer and W. Grimson. Learning patterns of activity using real-time tracking. IEEE Transactions on Pattern Analysis and Machine Intelligence, 22(8):747-757, August 2000. 1

[19] J. B. Tenenbaum. Mapping a manifold of perceptual observations. 1998. Advances in Neural Information Processing Systems 10, pages 682-688. 2

[20] J. M. Wang, D. Fleet, and A. Hertzmann. Gaussian process dynamical models for human motion. IEEE Transactions on Pattern Analysis and Machine Intelligence, 30(2):283-298, 2008. 2

[21] H. Zhong, J. Shi, and M. Visontai. Detecting unusual activity in video. In Proceedings of the IEEE Conference on Computer Vision and Pattern Recognition, volume 2, pages 819-826, Los Alamitos, CA, USA, 2004. 1 